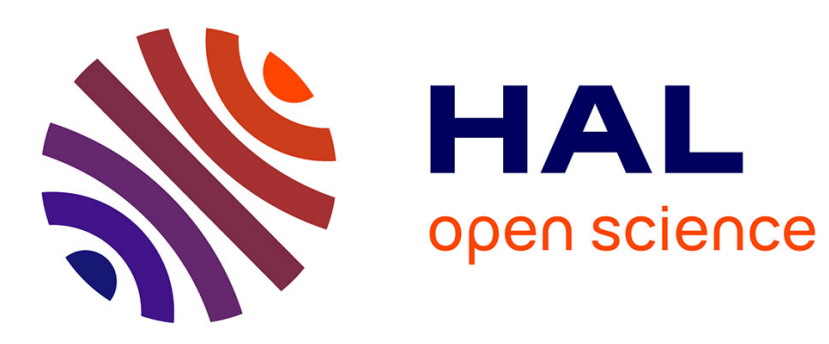

\title{
Using Xenopus Neural Crest Explants to Study Epithelial-Mesenchymal Transition
}

Nadège Gouignard, Christian Rouvière, Eric Theveneau

\section{To cite this version:}

Nadège Gouignard, Christian Rouvière, Eric Theveneau. Using Xenopus Neural Crest Explants to Study Epithelial-Mesenchymal Transition. The Epithelial-to Mesenchymal Transition, 2179, Springer US, pp.257-274, 2021, Methods in Molecular Biology, 10.1007/978-1-0716-0779-4_20 . hal-03365130

\section{HAL Id: hal-03365130 https://hal.science/hal-03365130}

Submitted on 17 Nov 2021

HAL is a multi-disciplinary open access archive for the deposit and dissemination of scientific research documents, whether they are published or not. The documents may come from teaching and research institutions in France or abroad, or from public or private research centers.
L'archive ouverte pluridisciplinaire HAL, est destinée au dépôt et à la diffusion de documents scientifiques de niveau recherche, publiés ou non, émanant des établissements d'enseignement et de recherche français ou étrangers, des laboratoires publics ou privés. 


\title{
Using Xenopus Neural Crest explants to study epithelial-mesenchymal transition
}

\author{
Nadège Gouignard ${ }^{1}$, Christian Rouvière ${ }^{1}$ and Eric Theveneau * \\ ${ }^{1}$ Centre de Biologie du Développement (CBD), Centre de Biologie Intégrative (CBI), \\ Université de Toulouse, CNRS, UPS, 118 route de Narbonne 31062, Toulouse Cedex \\ 09, France. \\ * eric.theveneau@univ-tlse3.fr
}

Running title: Using Xenopus Neural Crest to study EMT

Key words: neural crest, epithelial-mesenchymal transition, cell migration, adhesion, polarity, dispersion 


\section{Abstract}

The epithelial-mesenchymal transition (EMT) converts coherent epithelial structures into single cells. EMT is a dynamic cellular process that is not systematically completed (not all EMTs lead to single cells) and reversible (cells can re-epithelialize). EMT is orchestrated at multiple levels from transcription, to post-translational modifications, to protein turnover. It involves remodeling of polarity and adhesion and enhances migratory capabilities. During physiological events such as embryogenesis or wound healing EMT is used to initiate cell migration, but EMT can also occur in pathological settings. In particular, EMT has been linked to tissues fibrosis and cancer. Neural crest (NC) cells, an embryonic stem cell population whose behavior recapitulates the main steps of carcinoma progression, are a great model to study EMT. In this chapter, we provide a fully detailed protocol to extract NC cells from Xenopus embryos and culture them to study the dynamics of cell-cell adhesion, cell motility and dispersion. 


\section{Introduction}

In the 60's, during chick embryo gastrulation, epiblast cells were shown to dissociate from the epithelium, lose their epithelial morphology and become mesoderm and endoderm cells $[1,2]$. The concept of Epithelial-Mesenchymal Transformation was then introduced and defined as a complete loss of epithelial traits, including apico-basal polarity and cell-cell adhesion, accompanied by total acquisition of mesenchymal characteristics (e.g. front-back polarity, cell motility) [1,2]. E-Cadherin, a cell-cell junction protein, and vimentin, an intermediate filament protein, were soon proposed as makers for epithelial and mesenchymal cells respectively. However, a decade later, the term transition was preferred over transformation to reflect the dynamics, the complexity and the plasticity of this mechanism. Indeed, cells were described in various stages of EMT co-expressing markers of epithelial and mesenchymal states or neither. For a historical perspective on the field of EMT we recommend the review by Angela Nieto in Annual Reviews of Cell and Developmental Biology published in 2011 [3].

EMT can be initiated by various signaling pathways, including the Bone Morphogenetic Protein (BNP), Wnt, Fibroblast Growth Factor (FGF), Notch and hypoxia pathways, which induce the expression of transcription factors from the Twist, Snail, SoxE, FoxD, Ets and Zeb families. These transcription factors modulate the expression of the proteins involved in cell-cell junctions (type I and type II cadherins) to reduce cell-cell junction strength and promote the loss of apicobasal polarity. They also induce the expression of cell-matrix adhesion proteins (e.g. Integrins), extracellular matrix components (e.g. Fibronectin, Collagen, Vitronectin, Laminin) and remodeling factors (e.g. ADAMs, MMPs) as well as cytoskeleton proteins and regulators (e.g. Keratins, Vimentin, small GTPases) necessary for front-back polarity and motility. In the 90's, a parallel was drawn between tumor progression/metastasis and EMT, identifying common actors and signaling pathways (reviewed in [4,5]). It was suggested that cancer cells were hijacking the 
embryonic EMT programs. Therefore, a better understanding of the molecular mechanisms regulating EMT was believed to be of primary importance to understand tumor progression.

During the development of vertebrate embryos, EMT is essential for the delamination and long-distance migration of neural crest $(\mathrm{NC})$ cells, a transient multipotent population of cells that arises at the neural plate border and gives rise to a wide variety of derivatives [6]. Over the years, NC cells became a well establish model for physiological EMT, delamination and migration mechanisms, notably for its accessibility and ease of manipulation both in vivo and ex vivo.

In particular, Xenopus NC development recapitulates the main step of EMT and longdistance migration observed in most epithelial cancers. Xenopus NC cells can be defined as the cell population initiating the expressing of a repertoire of EMT transcription factors (Twist1, Snail1/2, Ets1, Zeb2) at the border of the neural plate, at the end of neurulation. Expression of these genes is controlled by the BMP, Wnt, FGF, Notch and hypoxia pathways [7-9]. Xenopus NC cells then undergo a typical case of cadherin switching, going from E-cadherin-dependent cell-cell junctions to $\mathrm{N}$-cadherin ones [10]. This switch is associated with a change of cell polarity from apico-basal, to front-rear, and is directly linked to the acquisition of motility and dispersion, by endowing NC cells with the ability to perform contact-inhibition of locomotion [10]. Importantly, EMT in Xenopus NC cells is also linked wtih the acquisition of chemotactic capabilities, since the Hif pathway co-regulates Twist, an E-cadherin repressor, and the expression of CXCR4, the main receptor for CXCL12/Stromal cell-derived factor 1 [9,11]. Finally, Xenopus NC cells express a wide range of matrix remodeling factors including several MMPs and ADAMs [12]. Altogether, this makes the Xenopus NC cells an extremely relevant model system to study EMT, from its upstream regulators to its downstream effectors and their impact on cell motility.

From a practical point of view, Xenopus produce a high number of large embryos, developing externally, allowing complete access at all times to developmental stages which are 
hardly accessible in other models. Furthermore, Xenopus embryos allow for gain and loss of function studies, by simple microinjection of Morpholino oligonucleotides, CRISPR/gRNA or mRNA in blastomeres during segmentation. The use of non-toxic fluorescent dyes permits lineage tracing during developmental processes. Using this technique, a cell fate map was generated, allowing reliable targeting of subpopulations of cells, including NC, by injecting specific blastomeres. Finally, NC are easily extracted from developing embryos to be grafted to host embryos for in vivo studes, or cultured ex-vivo in coated petri-dishes in a simple culture medium up to differentiation stages. If dissected at late neurula stage, NC are fully induced and will spontaneously undergo EMT-driven dispersion and migration during the first few hours following grafts or culture. EMT and migration of NC cells can be monitored in vivo or ex vivo using time-lapse cinematography.

In this book chapter, we describe the protocol to extract NC cells for ex vivo culture and discuss how to monitor and analyze NC dispersion and migration from time-lapse images.

\section{Materials}

\subsection{Ex-vivo culture of Xenopus Neural Crest cells}

\subsubsection{Extracellular matrix preparation}

1. Petri dishes (any dish from $35 \mathrm{~mm}$ to $90 \mathrm{~mm}$ made of untreated plastic) or multi-well dishes (e.g. LabTek, Ibidi $\mu$ Slides 80821 or equivalent).

2. Purified Fibronectin in solution (Sigma F1141 or equivalent reference from other providers).

3. Phosphate Buffer Saline (PBS) 1X: Start with $800 \mathrm{ml}$ of distilled water add $8 \mathrm{~g}$ of $\mathrm{NaCl}, 0.2 \mathrm{~g}$ of $\mathrm{KCl}, 1.44 \mathrm{~g}$ of $\mathrm{Na} 2 \mathrm{HPO} 4,0.24 \mathrm{~g}$ of $\mathrm{KH} 2 \mathrm{PO} 4$, adjust the $\mathrm{pH}$ to 7.4 with $\mathrm{HCl}$, add distilled water to a final volume of 1 liter. 
4. Bovine Serum Albumin (BSA).

5. Heating block.

\subsubsection{Microdissection (Fig. 1)}

1. Hair knife. Dip the tip of a short glass Pasteur pipette into melted wax and inset the root of a hair (eyebrow, eyelash or else) in the tip of the pipette before the wax has set.

2. A dish filled with modelling clay.

3. Glass ball. Prepared by melting the tip of a short glass Pasteur pipette.

4. Fine forceps.

5. P20 micropipette.

6. A small uncoated petri dish filled with culture medium to collect NC explants.

\subsubsection{Amphibian media and culture media}

1. Normal Amphibian Medium (NAM) stock solutions, must be autoclaved and can be stored at room temperature. In distilled water, $\mathrm{NAM} \mathrm{A}\left(\mathrm{NaCl}, 1.1 \mathrm{M} ; \mathrm{KCl}, 20 \mathrm{mM} ; \mathrm{NaHCO}_{3}, 10 \mathrm{mM}\right), \mathrm{NAM} B$ (MgSO4, 10mM; CaCl2, 10mM), NAM C (EDTA, $1 \mathrm{mM})$, NAM D $\left(\mathrm{Na}_{2} \mathrm{HPO}_{4}, 16 \mathrm{mM} ; \mathrm{NaH}_{2} \mathrm{PO}_{4}\right.$, $4 \mathrm{mM})$.

2. Working solutions: NAM $0.1 \mathrm{X}$ (for one liter add $10 \mathrm{~mL}$ of each NAM stock solutions and add $960 \mathrm{~mL}$ of distilled water), NAM $0.25 X$ (for one liter add $25 \mathrm{~mL}$ of each NAM stock solutions and add $900 \mathrm{~mL}$ of distilled water). 
3. Culture medium - Danilchik's for Amy (DFA): $53 \mathrm{mM} \mathrm{NaCl}, 5 \mathrm{mM} \mathrm{Na}_{2} \mathrm{CO}_{3}, 4.5 \mathrm{mM}$ KGluconate, $32 \mathrm{mM}$ NaGluconate, $1 \mathrm{mM} \mathrm{MgSO} 4\left(7 \mathrm{H}_{2} \mathrm{O}\right), 1 \mathrm{mM} \mathrm{CaCl}$, $0.1 \%$ BSA; adjust pH to 8.3 with $1 \mathrm{M}$ Bicine. Store at $-20^{\circ} \mathrm{C}$. After thawing, add $1000 \mathrm{U}$ penicillin and $100 \mu \mathrm{g} / \mathrm{ml}$ streptomycin.

\subsection{Time-lapse imaging}

1. Microscopes: inverted, upright microscope, dissecting microscope (see Note 1).

2. Camera and software.

3. Temperature controlled room (see Note 2).

4. Formaldehyde.

\subsection{Analysis of cell migration and dispersion dynamics}

1. A computer equipped with a modern CPU and with sufficient amount of RAM and storage space to process the data. The total RAM available should be twice the size of your largest dataset to allow opening and processing of the data.

2. A free (FIJl/Image J) or commercial software (e.g. Imaris/Bitplane) for automated detection of nuclei, tracking and dispersion analysis.

\section{Methods}

\subsection{Ex-vivo culture of Xenopus Neural Crest cells}

\subsubsection{Extracellular matrix preparation}


1. Aliquot the Fibronectin solution as a $10 \mathrm{X}$ stock by 20 or $50 \mu \mathrm{L}$ in $500 \mu \mathrm{L}$ centrifuge tubes and store at $-20^{\circ} \mathrm{C}$. For coating on glass coverslips, the $10 \mathrm{X}$ stock is at $1 \mathrm{mg} / \mathrm{mL}$. For coating on plastic petri dishes, the $10 X$ stock is at $100 \mu \mathrm{g} / \mathrm{mL}$. When making the $10 X$ stock for plastic, use PBS1X containing $0.1 \%$ BSA for long-term stability of the solution.

2. On the day of the experiment, select a dish appropriate to your microscope (see Note 3).

3. For glass dishes/coverslips dilute your Fibronectin in PBS1X to a final concentration of 100 $\mu \mathrm{g} / \mathrm{mL}$, for plastic, to $10 \mu \mathrm{g} / \mathrm{mL}$. Directly thaw the frozen aliquot of Fibronectin by adding the required amount of PBS1X at room temperature in the Fibronectin aliquot. (e.g. add $450 \mu \mathrm{L}$ of PBS1X on top of a $50 \mu \mathrm{L}$ aliquot of $10 \mathrm{X}$ Fibronectin to obtain $500 \mu \mathrm{L}$ of Fibronectin $1 \mathrm{X}$ for coating) (see Note 4).

4. Place the dish on a heating block for 1 hour at $37^{\circ} \mathrm{C}$.

5. Remove the fibronectin solution using a P1000 micropipette and rinse once with PBS1X (see Note 5).

6. Rinse the dish with a small amount of DFA1X to remove traces of PBS1X.

7. Fill the dish with DFA1X.

\subsubsection{Microdissection}

1. Place embryos at stage $15 / 16$ in a petri dish filled with NAM $0.25 \mathrm{X}$ and remove the vitelline membrane using fine forceps. Let them recover as superficial wounding may have occurred and let embryos further develop to stage 18 (see Note 6, Movie 1).

2. Select embryos at stage 18 (Movie 2). 
3. Fill the dish containing the modelling clay with NAM $0.25 \mathrm{X}$. Using the glass ball, form small holes in the modelling clay. The depth of each hole should be roughly half the width of an embryo (see Note 7, Movie 3).

4. Place one embryo per hole. Turn each embryo slightly on one side so that the neural crest region to be removed is facing up (Fig. 2a, Movie 3).

5. Using the glass ball, move gently the clay towards the embryo until it touches the epidermis making sure the orientation is preserved (Fig. 2b) then apply pressure all around the embryo by pushing the clay onto its sides to firmly secure the embryo (Fig. 2c, Movie 3, see Note 8).

6. Using the hair knife make a small incision posteriorly and dorsally to the neural crest area (Fig. 2c step 1, Movie 3).

7. Insert the tip of the hair knife in that initial hole, keeping the hair parallel to the tissue. The hair knife should be in between the pigmented layer and the neural tissue (Fig. 2c step 2, Movie 3)

8. Gently remove the pigmented layer from medial to lateral to uncover the neural crest region (Figure 2c step 3, Movie 3).

9. Place the hair knife parallel to the ventral side of the neural crest region (dotted line on Fig. 2d). Apply a gentle pressure (do not attempt to cut the tissue) and move from lateral to medial repeatedly. The neural crest region will detach from its surroundings (Fig. 2e-e'). Continue until the whole region detaches by itself (Movie 2). After dissection there should be a hole in which the underlying mesoderm can be seen (Figure 2f). When a dissected embryo is released from the clay the actual size of the wound should be relatively small and the neural tube on the dissected side should be intact (Figure 2g-g'). Look out for contamination from other cell types (Fig. 3, see Note 9). An example of a rough dissection including mesoderm is shown at the end of Movie 3. 
10. Using a P20 micropipette set to $10 \mu \mathrm{L}$ rapidly transfer the explant into a dish containing DFA1X (Movie 3).

11. Repeat steps 3 to 10 to collect multiple explants.

12. Cut the explants in small pieces using the hair knife and fine forceps (Movie 4). A complete unilateral neural crest region can be cut into 4 to 5 pieces each containing $\sim 100-200$ cells.

13. Transfer the explants onto the Fibronectin-coated dish.

14. Array the explants to facilitate monitoring of the explants under the microscope (see Note 10).

15. Let the explants adhere to the substrate for at least 15 minutes before moving the dish.

\subsection{Time-lapse imaging}

1. Gently place your dish onto the microscope.

2. Check explants viability and fluorescence if embryos were microinjected with a tracer prior to dissection.

3. Adjust lighting conditions (light intensity, exposure time).

4. Record the XY positions of each explants.

5. Set the time interval and total duration of the movie (see Note 11).

6. Start the acquisition.

7. At the end of the movie, cells can be fixed in formaldehyde $4 \%$ for further analysis and staining (see Note 12). Typical dispersion of control neural crest explants is shown in Movie 4 and Fig. 4. 


\subsection{Analysis of cell migration and dispersion dynamics}

\subsubsection{Analysis of cell migration}

1. A nuclear tracer (e.g. H2B-mCherry/GFP) must be injected into early Xenopus embryos prior to dissection if single cell tracking is to be performed (see Note 13). Manual or automated tracking can then be done using ImageJ/FIJI (Manual tracking or trackmate plug-in) or commercial alternatives such as Imaris/Bitplane (spot tracking algorithm).

2. From tracks, cell speed and directionality can be retrieved. This information can then be correlated with the dynamics of cell dispersion (see Note 14).

\subsubsection{Analysis of cell dispersion using the total explored area over time (Fig. 4)}

1. In ImageJ/FIJI use the "imagelstacks|tools|make substack" tool to extract images from each movie at regular interval (e.g. first image and then one image per hour). Save all substacks (one per $\mathrm{XY}$ position) into a separate folder for analysis (Fig. 4a).

2. In ImageJ/FIJI, link all external cells of an explant using the broken line tool and measure the area. Repeat this step for each time step of the substack (Fig. 4b-c). Repeat for each substack movie. Save the results as .xls or tabulated text file.

3. If only one experimental condition is analyzed over time, plotting the data as box whiskers plots for each time point will allow variability at each time point and overall dispersion over time to be assessed. If multiple conditions are to be plotted in parallel on the same graph, curves representing the mean area per time point alongside the standard deviation are more practical. 


\subsubsection{Analysis of cell dispersion using triangulation between nearest neighbors}

1. In ImageJ/FIJI use the "imagelstacks|tools|make substack" tool to extract images from each movie at regular interval (e.g. first image and then one image per hour). Save all substacks (one per $\mathrm{XY}$ position) into a separate folder for analysis.

2. In imageJ/FIJI, go to "analyze/set measurements" and make sure "centroid" and "area" are selected.

3. Open one of the substacks and estimate the mean area occupied by one nucleus in your samples by measuring about 20 different nuclei using the elliptical or polygon selection tools in ImageJ/FIJI.

4. Go to "analyze/analyze particles". Set the minimum and maximum area occupied by a nucleus as measured in step 3 and press OK. This will produce a list of $X Y$ coordinates of all nuclei detected.

5. Use the Delaunay/Voronoi plug-in in ImageJ/FIJI to build triangles from coordinates produced by the "analyze particles" tool. The options "infer selection from particles" and "make Delaunay ROI" must be selected.

6. Go to "analyze/tools/ROI manager" and do the following steps: "Add", "Split", "multimeasures".

7. Save the results table containing the areas of all triangles. It is important to note that the first value of that list is the cumulated area occupied by all triangles (see Note 15.)

8. If this has to be done on multiple images, a macro can be used. A macro for batch processing and a custom-made Look-up table (LUT) for color-coding of triangles were developed in the Theveneau lab and are available upon request. 
The respective merits of the two methods to analyze dispersion are discussed in Note 16.

\section{Notes}

1. In Xenopus NC cells the dynamics of dispersion depends on the timing of cell-cell dissociation, which is linked to the E-to-N-cadherin switch. Thus, the timing (how long it takes) and intensity (are all cells becoming individual?) of dispersion are a proxy for EMT dynamics. This can be assessed on low magnification images even without time-lapse if several conditions are plated at the same t0 and compared at a similar time point after the beginning of the cell culture. Therefore, monitoring simultaneously several explants at low magnification on a dissecting microscope could be sufficient for a first screening of experimental conditions. However, if dispersion analysis is to be performed using single cell detection by nuclear staining or if cell tracking is to be done in parallel, then higher magnifications (in $\mathrm{XY}$ and time) are required and a motorized stage is necessary. A wide field microscope with a 10X objective will allow visualization of NC explants the size of a fifth of a complete unilateral NC region, while providing enough $X Y$ resolution to track single cells accurately using a time interval of $3 \mathrm{~min}$. If cells are labelled with a membrane tracer and the dynamics of cell-cell contacts is to be analyzed, 20X or 40X objectives are needed and shorter time intervals are necessary (1 min or below). Inverted microscopes are the usual choice for cell culture assays. However, we obtained very good results using upright microscopes with dry lenses. In this case, cells need to be cultured in a dish that can be filled entirely with culture medium, without any air bubbles, and flipped upside down. Several providers have culture dishes with tight lids in their catalogue. If your cell culture device of choice does not exist in a version with a tight-fit lid, it is possible to seal the lid using high vacuum silicone grease. This type of upside-down cell culture removes all 
dead and/or non-adherent cells, keeping the substrate clean for the healthy cells. However, this set-up is not appropriate if experimental conditions impairs cell-matrix adhesion as cells would detach and thus be lost.

2. Xenopus embryos can develop between 12 and $28^{\circ} \mathrm{C}$, the optimal range being 14 to $25^{\circ} \mathrm{C}$. $\mathrm{NC}$ explants are a bit less flexible and usually survive and migrate better between 18 and $21^{\circ} \mathrm{C}$. Below $18^{\circ} \mathrm{C}$ cell-cell dispersion is affected and explants usually remain as pseudoepithelial sheets for much longer than they would in vivo. Above $21^{\circ} \mathrm{C}$, cell survival is lower. Between 18 $21^{\circ} \mathrm{C}$, explants roughly the size of a fifth of one unilateral NC region dissected at stage 18 spread extensively during the first two hours and then disperse as small clusters and eventually as single cells.

3. On upright microscopes, use tight-fit lid petri dishes that can be completely filled with culture medium and closed hermetically or open dishes with a water-immersion lens. For inverted microscopes, any type of multi-well dishes can be used depending on the type of motorized stage. In our hands, the 8-well Ibidi $\mu$ Slides (80821) gave the best results. They allow culture in small volumes $(300 \mu \mathrm{L})$ suitable for drug screening. They are made of a polymer that allows low concentration of Fibronectin coating for plastic to be used while being compatible with confocal imaging.

4. When coating large dishes do not cover the entire dish with Fibronectin. Use a marker pen to delimitate an area where Fibronectin will be coated. A circle with a diameter of $1 \mathrm{~cm}$ is enough to plate 5 rows of 5 explants and can be coated with a $500 \mu \mathrm{L}$ drop of Fibronectin $1 \mathrm{X}$ solution. Always cover the dish during Fibronectin incubation at $37^{\circ} \mathrm{C}$ to avoid evaporation. If very large dishes are used (e.g. $90 \mathrm{~mm}$ bacteria plates), place a piece of wet paper inside the dish to keep it moist. 
5. A successful Fibronectin coating can be assessed by eye. When the Fibronectin solution is removed at the end of the 1 -hour incubation, the part of the dish or the well that is coated remains slightly wet and appears shiny when exposed to light whereas a similar incubation with a drop of water or PBS1X will leave the dish dry as soon as the liquid is removed. The area that received water or PBS cannot be distinguished from the rest of the dish.

6. Dissection of Xenopus NC explants is very easy when the embryos are at the right developmental stage. Just prior to migration, NC cells are not significantly attached to their surrounding tissues (ectoderm, mesoderm, ectodermal placodes). Yet, they strongly attach to each other. This allows the whole NC region to be removed, as it is an epithelial structure only loosely connected to the other tissues. The best stage is 18 . At this stage the neural folds nearly finished closing (Movie 2). Only the most anterior part of the neural plate is still open and has the shape of a tear drop. The NC region appears as a bump lateral to the anterior neural plate and extends roughly to the middle of the anteroposterior axis. Before that stage, the NC region is still attached to the neural plate and one would need to arbitrarily cut the explant out of the embryo without any clear landmark to use as reference. After stage 18 , cells initiate migration and start adhering strongly to the mesoderm and the ectoderm. First, many cells are lost while peeling the superficial ectoderm. Second, it increases the probability of mesoderm contamination (Fig. 3, Fig. 5, last part of Movie 3). Dissections at early (prior to stage 18) or late stages (after stage 18) are still technically possible, but they require an extensive amount of training. If such stages are absolutely needed for a given project, one should train dissecting by placing embryos at similar stages that have been stained with a neural crest marker (e.g. slug, twist) next to the embryos to be dissected, for reference.

7. Embryos should not be put completely inside the clay. It is important that roughly a third of the embryo still pops out of the modelling clay after the embryo has been immobilized. Otherwise, the region of interest will be difficult to access for dissection with the hair knife. 
8. Once the embryo has been immobilized by applying pressure with the modelling clay, all previous morphological landmarks are gone. Thus, it is critical that the orientation of the embryo to be dissected is correct before applying pressure.

9. At the end of neurulation (stage 18), NC cells adhere to each other more than they adhere to their surrounding tissues (see Note 6) making dissection relatively easy. Yet, contamination from adjacent cell types can sometimes occur. Mesoderm and endoderm are richer in vitellus and appear whiter than NC cells, which are comparatively translucent and grey. The last part of Movie 3 shows an example of a rough dissection during which a bit of mesoderm is taken out together with the neural crest. See also Fig. 3 and its legend to see how to proceed when mesoderm is mistakenly dissected alongside NC cells. Superficial ectoderm may be detected by the presence of dark spots corresponding to the pigments. Explants should always be screened for contamination at the end of the culture and explants with contamination from other cell types should be excluded. In 2D-culture, ectoderm, neural plate, neural crest, mesoderm and endoderm can be easily recognized morphologically using several criteria such as the shape of the cells and nuclei or the size of vitellus platelets (Fig. 5). Non-neural ectodermal cells flatten extensively onto the substrate and form a continuous monolayers with large nuclei. Neural plate explants remain as a tight cluster and project axons within few hours of cell culture. Mesodermal and endodermal cells are significantly larger and whiter than neural crest cells. They contain large vitelline platelets.

10. When plating explants next to each other onto Fibronectin, do not put explants to close to one another as NC cells can attract each other via a process called co-attraction or mutual attraction. This would lead neighboring explants to merge. A rule of thumb is to make sure that each explant is at least at 5 times its own diameter away from the nearest explants in the dish.

11. If only overall explant dispersion is to be analyzed, time-lapse imaging is not necessary. Explants can be fixed after several hours and the dispersion can be analyzed using triangulation, 
total explant area or nearest neighbors analysis. However, having the dynamics of the population and the movement of single cells can be very informative. For movies done with a 10X objective at a temperature between $18-21^{\circ} \mathrm{C}$, cells migrate at around $1-3 \mu \mathrm{m} / \mathrm{min}$ and a time interval of 3 minutes is optimal for cell tracking (either manual or automated). 8-hour movies at $21^{\circ} \mathrm{C}$ are enough to see the main steps of dispersion: adhesion, spreading, break-up of the explants as clusters and single cell dispersion. If under control conditions single cell dispersion does not occur within this time frame it can be due to several possibilities: the temperature is too low, cells were extracted at earlier stages than 18 and have not yet completed their cadherin switch (there is a sharp decrease of the amount of E-cadherin from stage 15), the substrate is not optimal for migration and cells have problems moving away from each other.

12. To fix cells at the end of a culture experiments do not remove the DFA1X, instead add directly formaldehyde into the dish. In 8-well slides, if each well contains $300 \mu \mathrm{L}$ of DFA1X, simply add $100 \mu \mathrm{L}$ of formaldehyde $16 \%$ in each well. In larger dishes, add locally formaldehyde $16 \%$ atop the explants to pre-fix the cells, wait 5 minutes, remove the medium, fill with formaldehyde $4 \%$ and further fix for 30 minutes.

13. To label NC cells prior to cell culture, one can inject at either $2,4,8,16$ or 32 -cell stage. If simple tracers to label nuclei and/or membranes are used, the easiest way is to inject two blastomeres at 2-cell stage and select the brightest embryos at stage 16 prior to removing the vitelline membrane. If nuclear/membrane tracers are to be used in combination with other treatments that may affect early development, it is recommended to inject at 8-cell stage or later. From 8-cell stage the animal blastomeres only contribute to the ectoderm (neural plate, NC, placodes, epidermis), therefore injections at 8-cell stage or later will leave the mesoderm and endoderm intact, reducing possible side effects on gastrulation.

14. In control conditions, cell-cell dissociation leads to a progressive shift from collective/pseudoepithelial-like behavior, to a collective/mesenchymal behavior, to single cell 
behavior. Individual cell directionality decreases in single cells compared to cells within a group, whereas individual cell speed increases. This, in control conditions, typically correlates with the rate of expansion of the explant. The initial slow-paced directional radial expansion turns into a fast disorganized dispersion of single cells. Overall dispersion comes to a halt when local cell density is such that cells have an equal probability of moving in any direction. However, under experimental conditions, one can interfere with cell-cell or cell-matrix adhesion and affect the relationship between explant dispersion and single cell behavior. For instance, reducing cell-cell adhesion may promote single cell behavior while overall radial expansion of the explant is impaired. Thus analyzing single cell behavior and explant dispersion in parallel can be very informative.

15. Triangles' areas follow an exponential distribution, thus the usual statistical tests for normal/Gaussian datasets do not apply if one wants to compare the distribution of triangles' areas from one explant to another. This problem is easily solved when comparing populations of explants. One should plot the mean triangle area of each explant per experimental condition. That way each explant has the same weight in the mean of a given condition (otherwise explants with more cells contribute more triangles and can skew the mean of the dataset). In addition, the distribution of mean areas under control condition follows a Gaussian distribution and allows simple statistical tests to be performed.

16. Analyzing explant dispersion using the total explored area is the simplest and most effective way. This technique does not require pre- or post- labelling of cell's nuclei. It enables the inclusion of the impact of all cells, and can be done on simple low resolution bright field or phase contrast images. Triangulation on the other hand has several caveats. First, one needs to detect single cell nuclei. This can be done by labelling NC cells with a nuclear tracer or post-staining them with DAPI. Automatic detection of nuclei is possible (e.g. analyze particles plug-in in Image $\mathrm{J} / \mathrm{FIJI}$ ) but weaker nuclei are often not detected, artificially increasing the area of some triangles. 
Similarly, areas with high cell density cannot be properly segmented and this results in artefacts (Fig. 4). This later problem can be partially solved by acquiring images on a confocal microscope, although high resolution images of nuclei often confuses the automated detection of particles, since pixel intensity within each nucleus can vary substantially, and nuclei no longer appear as solid structures. Alternatively, one can mark each cell's nucleus manually. The first option, using confocal imaging, will never be a realistic routine procedure, even if one owns a dedicated confocal microscope for time-lapse imaging. The second option, manual marking of individual nuclei, is extremely time consuming, especially if one wants to perform triangulation at multiple time points for multiple explants to assess the dynamics of dispersion. A typical explant has between 100 and 200 cells. If one wants to perform triangulation on 20 explants at 5 time points each, it means marking manually between 10000-20000 nuclei. This increases exponentially if one needs to do experiments in triplicate and has to compare multiple experimental conditions. Thus, if one absolutely needs to use triangulation (e.g. local differences in terms of distance between neighbors within each explant are important), we recommend using the automated detection of nuclei. However, it is important to bear in mind that some nuclei will not be detected regardless of the technique used to mark them, or to detect them. It is important to first establish the conditions for automated detection on a small subset of data and to compare the overall output with the one obtained when marking nuclei manually. A difference of more than $5 \%$ should not be considered acceptable and image acquisition and nuclei detection would need to be improved before embarking on large scale automated detection of nuclei.

\section{References}

\section{Acknowledgements}


Eric Theveneau and Christian Rouviere are permanent CNRS staff. Work in the Theveneau lab is supported by the Region Midi-Pyrenees (Installation Grants for Excellent Researchers, 13053025), the Fondation pour la Recherche Medicale (AJE201224), the CNRS and the Université Paul Sabatier. Nadège Gouignard is the recipient of an individual fellowship from FRM (ARF20150934153) and the Marie Curie Prestiges Program (PRESTIGES 2015-4-007).

\section{Figures Legends}

\section{Figure 1 - Tools for microdissection of Neural Crest explants}

a, Tools for microdissection: from left to right, fine forceps to remove the vitelline membrane, plastic Pasteur pipette for transferring embryos, hair knives and glass ball for digging holes in the modelling clay. b, dish filled with modelling clay to hold embryos during dissection. c, stereomicroscope. d, zoom-in on the tips of the hair knives. e, zoom-in on the tip of the glass ball. $f$, a small dish containing DFA1X to collect explants. $g$, a fibronectin-coated dish to culture the explants. All scale bars are $1 \mathrm{~cm}$, except in $\mathrm{d}$ and e, $500 \mu \mathrm{m}$.

\section{Figure 2 - Microdissection of neural crest cells: an ideal case.}

a, Stage 18 embryo slightly turned on its left side to place the right NC region up for dissection.

b, The clay has been gently pushed to make contact with the embryo and to lock the orientation.

c, The clay was further pushed towards the embryo to firmly maintain the embryo prior to dissection. 3 steps are necessary to remove the overlying pigmented layer. Step 1, make a superficial incision using the tip of the hair knife. Step 2, insert the hair knife underneath the skin, parallel to the embryo and move anteriorly. Do not stab the embryo towards the inside. Step 3, gently move from medial to lateral to remove the pigmented layer. Avoid using the tip of the hair 
knife, rather work with the side of the hair. $d$, The embryo after the superficial pigmented layer has been removed. The green dotted line indicates where the hair knife should be positioned to remove the neural crest. Slightly press down using the side of the hair knife and move from lateral to medial (step 4, green arrows). e, The NC explant (nc) has been lifted, the neural tube (nt) and mesoderm (m) are visible, see the zoom-in in e'. f, the NC explant has been removed, seen in f', the dissected region is shown in f'. $g-g$ ', the embryo after dissection and freed from the clay. Scale bars: a-g, $1 \mathrm{~mm}$; e', f' and g', $150 \mu \mathrm{m} ; \mathrm{f}^{\prime}, 500 \mu \mathrm{m}$.

Figure 3 - Microdissection of neural crest cells: how to handle mesoderm contamination.

a, Stage 18 embryo after the superficial pigmented layer has been removed. b, The NC explant was removed by going too deep within the tissue and a piece of mesoderm $(\mathrm{m})$ is coming with it. The endoderm (end) lining the internal cavity is visible. b', Zoom of the mesoderm contaminated NC explant. The dotted line delineates the mesoderm which appears whiter than the neural crest. b", Zoom of the endoderm at the site of the dissection. c, most of the mesoderm can be removed using the hair knife prior to detaching the neural crest explant but some mesoderm may remain ( $m$, arrow). $d, N C$ explant after being detached from the embryo, remaining mesoderm contamination is indicated by the arrows. e-g, Trying to clean the small bits of mesoderm is too difficult. Instead, cut the neural crest explant in two pieces and discard the half containing the mesoderm contamination. The explant on the right-hand side of panel $\mathrm{g}$ is good for culture, the one on the left-hand side should be discarded.

\section{Figure 4 - Analyzing the dynamics of cell dispersion}

a, Workflow from cell culture to substacks for dispersion analyses. b-c, Total explored area is retrieved by linking all external cells per explant, per time point. d-f, Triangulation analysis. d, 
thresholded image of DAPI staining. e, triangles obtained after performing particles detection and Delaunay/Voronoi triangulation. Each triangle is color-coded according to the following ratio (area of the triangle/total area). $\mathrm{f}$, example of artefacts in areas of high cell density due to poor particle detection. Steps on panels $d$ and e are repeated for each time point of interest.

Figure 5 - How to distinguish neural tube, skin, placodes and mesoderm from neural crest cells

a, Typical NC culture observed using bright field. After a few hours, the explant should have flattened on the substrate and cells should start dispersing. NC cells have small vitellus platelets and their nuclei should be visible as a translucent circle. b, Neural plate/tube explants do not flatten on the substrate. They form a solid block from which few cells may migrate out. Often axons are projected directly from the explant onto the matrix. c, Non-neural ectoderm and placodes. Even if the superficial pigmented layer has been properly removed the deep layers of the ectoderm are still present and may be taken out together with neural crest. The deep layers of the ectoderm surrounding the NC contain prospective skin cells and placodes. Skin cells flatten even more than NC and as a consequence their nuclei appear very large. They form a coherent sheet without any visible gaps. Placodes produces neurons of the cranial ganglia; therefore, placode contamination often leads to few grapes of round cells projecting axons after an overnight culture. d, Mesoderm. Mesodermal cells are rich in vitellus and the vitelline platelets are larger than those observed in crest cells. Also, the mesoderm underlying the neural crest at stage 18 flattens on the substrate but does not disperse extensively and rarely produces single cells.

\section{Movies}


Movie 1 - Removing the vitelline membrane of stage 16 Xenopus embryos

Movie 2 - Neurulation: how to identify stage $18 ?$

Movie 3 - Dissecting neural crest cells from stage 18 embryos

Movie 4 - Examples of control ex vivo neural crest cell migration

\section{References}

1. Hay ED (1995) An overview of epithelio-mesenchymal transformation. Acta anatomica 154 (1):8-20. doi:10.1159/000147748

2. Hay ED, Zuk A (1995) Transformations between epithelium and mesenchyme: normal, pathological, and experimentally induced. Am J Kidney Dis 26 (4):678-690. doi:10.1016/0272-6386(95)90610-x 3. Nieto MA (2011) The ins and outs of the epithelial to mesenchymal transition in health and disease. Annual review of cell and developmental biology 27:347-376. doi:10.1146/annurev-cellbio-092910154036

4. Thiery JP, Acloque $H$, Huang RY, Nieto MA (2009) Epithelial-mesenchymal transitions in development and disease. Cell 139 (5):871-890. doi:10.1016/j.cell.2009.11.007

5. Thiery JP (2002) Epithelial-mesenchymal transitions in tumour progression. Nature reviews Cancer 2 (6):442-454. doi:10.1038/nrc822

6. Gouignard N, Andrieu C, Theveneau E (2018) Neural crest delamination and migration: Looking forward to the next 150 years. Genesis:e23107. doi:10.1002/dvg.23107

7. Steventon B, Carmona-Fontaine C, Mayor R (2005) Genetic network during neural crest induction: from cell specification to cell survival. Seminars in cell \& developmental biology 16 (6):647-654. doi:10.1016/j.semcdb.2005.06.001

8. Steventon B, Mayor R (2012) Early neural crest induction requires an initial inhibition of Wnt signals. Developmental biology 365 (1):196-207. doi:10.1016/j.ydbio.2012.02.029

9. Barriga EH, Maxwell PH, Reyes AE, Mayor R (2013) The hypoxia factor Hif-1alpha controls neural crest chemotaxis and epithelial to mesenchymal transition. The Journal of cell biology 201 (5):759-776. doi:10.1083/jcb.201212100

10. Scarpa E, Szabo A, Bibonne A, Theveneau E, Parsons M, Mayor R (2015) Cadherin Switch during EMT in Neural Crest Cells Leads to Contact Inhibition of Locomotion via Repolarization of Forces.

Developmental cell 34 (4):421-434. doi:10.1016/j.devcel.2015.06.012

11. Theveneau E, Marchant L, Kuriyama S, Gull M, Moepps B, Parsons M, Mayor R (2010) Collective chemotaxis requires contact-dependent cell polarity. Developmental cell 19 (1):39-53.

doi:10.1016/j.devcel.2010.06.012

12. Christian L, Bahudhanapati H, Wei S (2013) Extracellular metalloproteinases in neural crest development and craniofacial morphogenesis. Critical reviews in biochemistry and molecular biology 48 (6):544-560. doi:10.3109/10409238.2013.838203 
